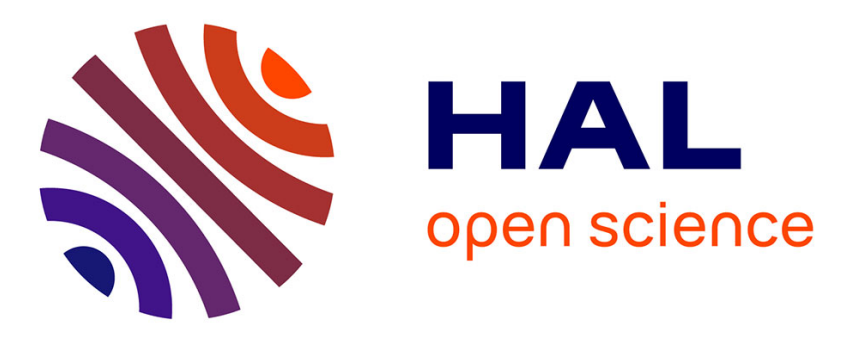

\title{
A new algorithm for spatiotemporal analysis of brain functional connectivity.
}

\author{
Ahmad Mheich, M Hassan, M Khalil, C Berrou, F Wendling
}

\section{To cite this version:}

Ahmad Mheich, M Hassan, M Khalil, C Berrou, F Wendling. A new algorithm for spatiotemporal analysis of brain functional connectivity.. Journal of Neuroscience Methods, 2015, 242C, pp.77-81. hal-01118260

\author{
HAL Id: hal-01118260 \\ https://hal.science/hal-01118260
}

Submitted on 18 Feb 2015

HAL is a multi-disciplinary open access archive for the deposit and dissemination of scientific research documents, whether they are published or not. The documents may come from teaching and research institutions in France or abroad, or from public or private research centers.
L'archive ouverte pluridisciplinaire HAL, est destinée au dépôt et à la diffusion de documents scientifiques de niveau recherche, publiés ou non, émanant des établissements d'enseignement et de recherche français ou étrangers, des laboratoires publics ou privés. 


\title{
A new algorithm for spatiotemporal analysis of brain functional connectivity
}

\author{
Mheich A. ${ }^{1,2,3}$, Hassan M. ${ }^{1,3}$, Khalil M. ${ }^{2}$, Berrou C. ${ }^{4}$ and Wendling F. ${ }^{1,3}$ \\ ${ }^{1}$ INSERM, U1099, Rennes, F-35000, France \\ ${ }^{2}$ AZM center-EDST, Lebanese University, Tripoli, Lebanon \\ ${ }^{3}$ Université de Rennes 1, LTSI, F-35000, France \\ ${ }^{4}$ Télécom Bretagne (Institut Mines-Télécom), UMR CNRS Lab-STICC, Brest, France
}

\section{Abstract}

Specific networks of interacting neuronal assemblies distributed within and across distinct brain regions underlie brain functions. In most cognitive tasks, these interactions are dynamic and take place at the millisecond time scale. Among neuroimaging techniques, Magneto/ Electroencephalography -M/EEG- allows for detection of very short-duration events and offers the single opportunity to follow, in time, the dynamic properties of cognitive processes (sub-millisecond temporal resolution).

In this paper we propose a new algorithm to track the functional brain connectivity dynamics. During a picture naming task, this algorithm aims at segmenting high-resolution EEG signals (hr-EEG) into functional connectivity microstates. The proposed algorithm is based on the K-means clustering of the connectivity graphs obtained from the Phase Locking Value (PLV) method applied on hr-EEG. Results show that the analyzed evoked responses can be divided into six clusters representing distinct networks sequentially involved during the cognitive task, from the picture presentation and recognition to the motor response. 


\section{Introduction}

There is increasing evidence that cognitive functions arise from the activation of networks distributed over distinct and possibly distant brain regions as opposed to isolated focal areas (Sporns 2010). Hence, efforts focused on the analysis of brain connectivity as a key concept to understand brain cognitive functions. Due to its excellent spatial resolution, fMRI has become one of the most commonly used noninvasive methods to study cerebral functions (Allen, Damaraju et al. 2012).

However, in many cases, the short duration of most cognitive processes $(\sim 500 \mathrm{~ms}$ for picture naming, for example) would greatly benefit from the use of techniques that have a much higher time resolution (on the order of $m s$ ), which is not the case of fMRI $(\sim 1 s)$. Along this line, several studies indicated that the use of electroencephalography (EEG, $1 \mathrm{~ms}$ time resolution for signals classically sampled at $1 \mathrm{kHz}$ ) combined with appropriate signal processing techniques can bring relevant information about normal networks during cognitive activity (Rodriguez, George et al. 1999) or about altered networks associated with tumors (Bartolomei, Bosma et al. 2006) for instance.

This excellent temporal resolution of the EEG signals allowed us to analyze the dynamic properties of cognitive processes, an issue so far addressed in a few studies only. In (Murray, Brunet et al. 2008), authors proposed an algorithm based on the amplitude of Event Related Potentials (ERPs) to follow time-varying voltage topographic maps. However, these algorithms do not account for brain connectivity quantified directly from scalp signals (electrode space) or indirectly from reconstructed brain sources (source space).

Regarding the approaches based on the connectivity analysis, most of reported methods make use of a constant time window to track the dynamics of functional connectivity, as estimated from EEG recordings. This window is typically chosen either empirically or based on a priori 
information about the analyzed task (Rodriguez, George et al. 1999). A few attempts have been recently reported to avoid this constraint (De Vico Fallani, Astolfi et al. 2008; Dimitriadis, Laskaris et al. 2010; Allen, Damaraju et al. 2012). However, most of proposed algorithms are not adapted to tracking changes over very short durations (in the order of 500 $\mathrm{ms}$, as in the case of responses evoked by visual stimuli).

In this paper, we propose a novel algorithm to track the dynamics of brain functional connectivity at millisecond scale. The proposed algorithm is based on the K-means clustering of the connectivity networks obtained by the Phase Locking Value (PLV) method. Performance evaluation was assessed on high-resolution electroencephalographic (hr-EEG) signals recorded in subjects during a picture naming task.

\section{Materials and Methods}

\section{A. Functional connectivity measure}

Functional connectivity is classically defined as the temporal correlation (wide sense) among electrophysiological signals generated by distinct neuronal assemblies (Friston 1994). Several methods have been proposed to quantify brain functional connectivity. In this study we used a method, which belongs to the so-called "phase synchronization" (PS) family.

It is well known that the respective phases of two oscillators may synchronize even if their amplitudes stay uncorrelated. The general principle of PS methods is to detect the existence of a phase locking between two systems defined as:

$$
\varphi_{x y}(t)=\left|\Phi_{x}(t)-\Phi_{y}(t)\right| \leq C
$$

where $\Phi_{x}(t)$ and $\Phi_{y}(t)$ are the unwrapped phases of the signals ( $x$ and $\left.y\right)$ representative of the two systems at time $t$ and $C$ a constant. The first step for estimating the phase synchronization is to extract the instantaneous phase of each signal. In this study, we used the method based 
on Hilbert transform. The second step is the definition of an appropriate index to measure the degree of synchronization between estimated instantaneous phases. To proceed, we used the phase locking value (PLV) (Lachaux, Rodriguez et al. 1999), as illustrated in figure 1-B. For each channel pair, $x$ and $y$, at time $t\left(t=t_{1}, . ., t_{T}\right.$ where $T=D * f_{\mathrm{s}} ; D$ and $f_{\mathrm{s}}$ denote the signal length relative to the onset and the sampling frequency, respectively) for the $N$ trials and for subject $j(j=1 \ldots M$, where $M$ denotes the number of subjects), PLV is defined as:

$$
\operatorname{PLV}_{x y}^{j}(t)=\frac{1}{N}\left|\sum_{n=1}^{N} \varphi_{x}(t)-\varphi_{y}(t)\right|
$$

To reduce the effect of correlations between near electrodes, we apply a normalization procedure (z-score) so that the PLV values were compared with the $200 \mathrm{~ms}$ baseline preceding the presentation of the image. Let $\mu_{x y}$ and $\sigma_{x y}$ are the mean and standard deviation computed from a $200 \mathrm{~ms}$ pre-stimulus baseline. The normalized PLVs are then defined as $\overline{\operatorname{PLV}}_{x y}^{j}(t)=\left(\operatorname{PLV}_{x y}^{j}(t)-\mu_{x y}^{j}\right) / \sigma_{x y}^{j}$. A thresholding procedure is then applied on the functional connectivity values in order to retain the strongest functional connections. The connectivity measure was computed in the low gamma frequency band $(30 \mathrm{~Hz}-45 \mathrm{~Hz})$. More precisely, the phases were estimated for each frequency and the average phase at $30-45 \mathrm{~Hz}$ was used. Indeed, this frequency band was shown to be highly relevant in the context of the cognitive task performed by subjects, as reported in (Rodriguez, George et al. 1999).

The PLVs were then averaged over subjects:

$$
\overline{\operatorname{PLV}}_{x y}(t)=\frac{1}{M} \sum_{j=1}^{M} \operatorname{PLV}_{x y}^{\mathrm{j}}(t)
$$

Where $\overline{\operatorname{PLV}}_{x y}(t)$ represents the general term of the average adjacency matrix $\overline{\operatorname{PLV}}(t)$ which defines a functional connectivity graph $\mathrm{G}$ at each time $t, G=\{G(t), t=1, . ., T\}$, computed for the $V$ pairs of $x$ and $y$ channels, where $V$ is equal to $(N c .(N c-1) / 2))$ and $N c$ is the number of 
channels in the hr-EEG montage. According to equations 1 and 2, $T$ adjacency matrices are obtained.

\section{B. Segmentation algorithm}

The objective of this algorithm is to identify clusters among the $T$ graphs $G(t)$. As illustrated in figure 1-C, the proposed algorithm is based on three main steps:

\section{Step 1: Initialization}

To start with, $K$ graphs $G^{k}, G^{k}=\left\{\bar{G}^{k}, k=t_{1}, . ., t_{K}\right\}$, are selected where $k=t_{l}$ and $l$ is randomly chosen in ( $K$ varies from 3 to 12 and $k$ varies from 1 to $K$ ) with the restriction of rejecting the $K$ graphs if the time interval between two $t_{l}$ is less than $30 \mathrm{~ms}$.

\section{Step 2: Assignment}

The spatial correlation $s C^{k}(t)$ between $G(t)$ and $\overline{G^{k}}$ is then computed as follows:

$$
\mathrm{sC}^{k}(t)=\frac{\sum_{i=1}^{V} \overline{G_{i}^{k}} \cdot G_{i}(t)}{\sqrt{\sum_{i=1}^{V} \overline{G_{i}^{k}}} \cdot \sqrt{\sum_{i=1}^{V} G_{i}^{2}(t)}}
$$

where $i$ denotes the $i^{\text {th }}$ edge in $G(t)$ and $\overline{G^{k}}$. As depicted in eq.3, sC is normalized by the variance of graphs $\mathrm{G}$ and $\mathrm{Gk}$. Thus, $\mathrm{sC}$ ranges from 0 to $1 \mathrm{High}$ values denote graph with high similarity. Conversely, low values are indicative of low similarity between graphs. 


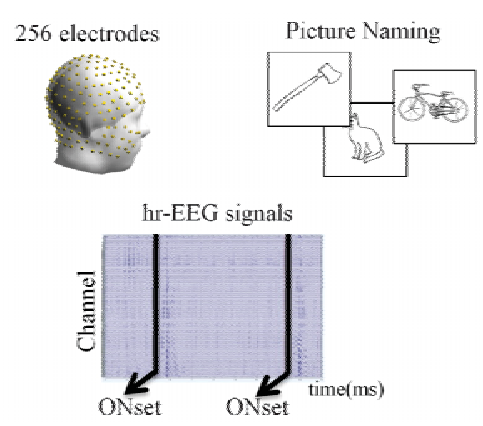

B

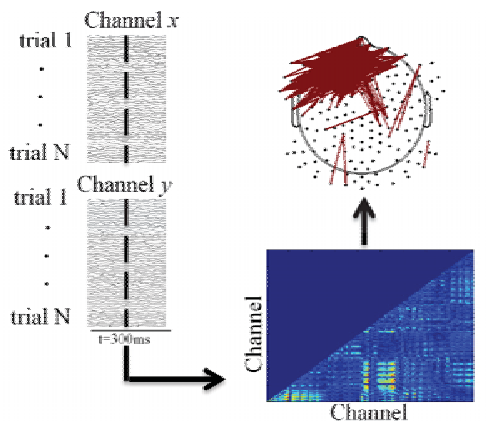

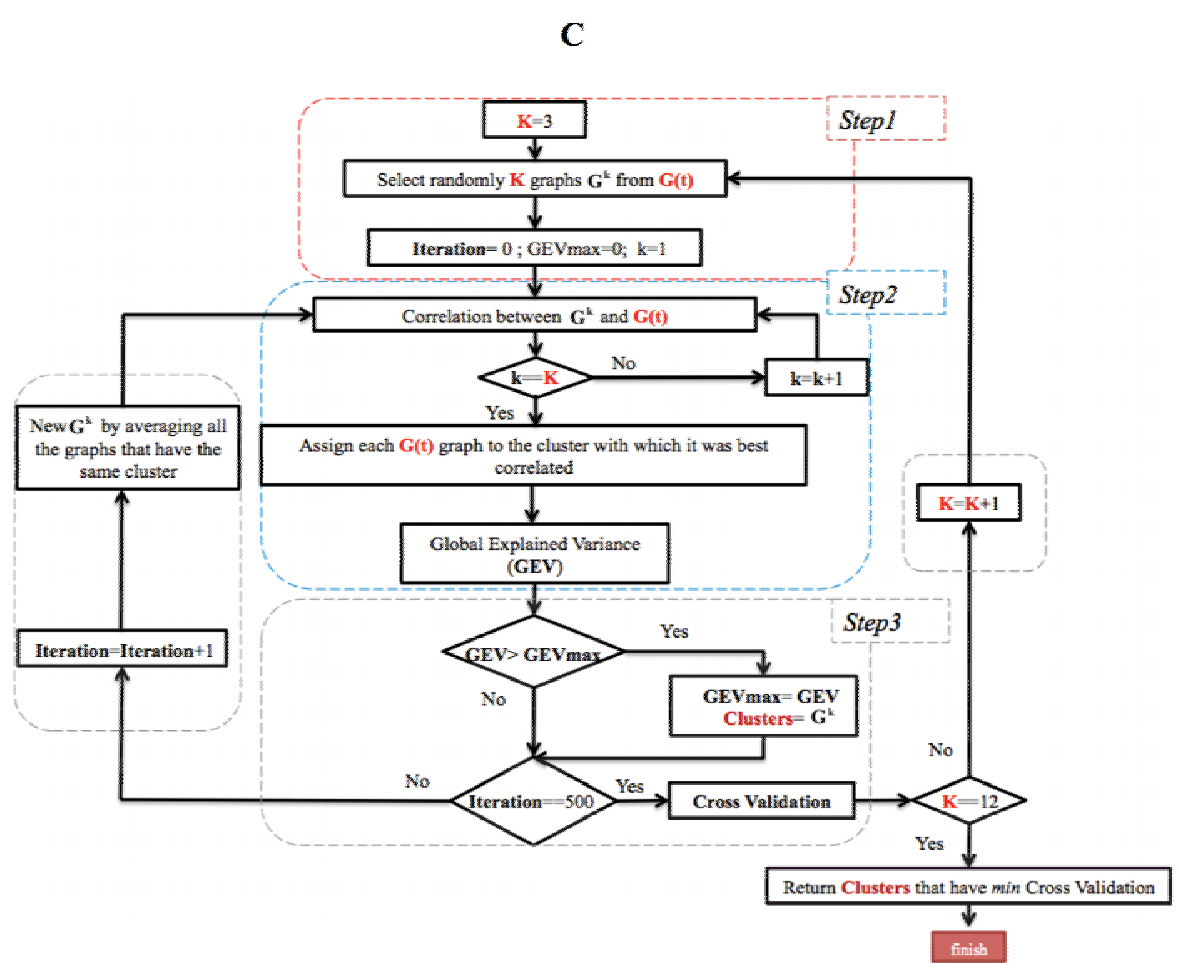

Figure 1: Structure of the investigation: A- Dense EEGs with 256 electrodes were used to record brain electrical activity. Participants were shown about 74 stimuli from different categories. B- Functional connectivity was computed between electrodes over all trials using the phase locking value method; the resultant connectivity matrix at a given instant (300ms for instance) was derived. This connectivity matrix was then projected on $2 \mathrm{D}$ scalp to provide a connectivity graph. C- Illustration of flowchart of the proposed segmentation algorithm: three main steps were realized: the initialization, the assignment and the update steps. 
Each graph $G(t)$ is then assigned to the cluster for which the spatial correlation was the highest. The assigned clusters are defined as $\hat{G}^{k}$ :

$$
\hat{G}^{k}=\left\{G(t): s C_{G(t), G^{k}}^{k} \geq s C_{G(t), G^{k^{\prime}}}^{k^{\prime}} \forall 1 \leq k^{\prime} \leq K\right\}
$$

From these spatial correlation values, the Global Explained Variance (GEV) is calculated as defined in (Murray, Brunet et al. 2008):

$$
\begin{aligned}
& G E V=\sum_{k=1}^{K} G E V^{k} \\
& G E V^{k}=\sum_{t=1}^{T}\left(s C_{G(t), G^{k}}\right)^{2} \cdot \gamma_{G(t), \hat{G}^{k}} \text { where } \gamma_{G(t), \hat{G}^{k}}=\begin{array}{l}
1 \text { if } G(t) \in \hat{G}^{k} \\
0 \text { if } G(t) \notin \hat{G}^{k}
\end{array}
\end{aligned}
$$

\section{Step 3: Update}

At each iteration, the new centroids $\overline{G^{k}}$ are updated by averaging all the graphs yielding to the same cluster

$$
\overline{G^{k}}=\frac{1}{\left|\hat{G}^{k}\right|} \sum_{G^{\prime} \in \hat{G}^{k}} G^{\prime}
$$

For each $K$, steps 2 and 3 were repeated 500 times. The set of centroids leading to the highest GEV was retained. When the algorithm converges (reaching the highest GEV), $K+1$ graphs $\bar{G}$ are then selected randomly and the entire above procedure (from step 2 to step 3 ) is repeated until $K=12$.

To choose the optimal number of clusters, we used a method based on the Cross Validation (CV) criterion (Murray, Brunet et al. 2008) which is a ratio between the GEV and the degrees of freedom for a given set of graphs. As reported, the global minimum of this criterion gives 
the optimal number of segments. Note that in the same segment, the graphs can have different $\mathrm{SC}$ values with the same cluster and therefore two consecutive graphs (in time) can be classified in two distinct clusters. To overcome this, the decision that is made is to choosing the 'most dominant' Cluster inside each segment.

Finally, the above-described method is a group-averaged approach means that this method was based on the computation of the spatial correlation between graphs on averaged $\overline{\operatorname{PLV}}(\mathrm{t})$ adjacency matrices obtained from all subjects. Nevertheless, the algorithm does not ignore the inter-subject variability. To analyze the single subject contribution, the same type of calculation (mainly step 2) can be performed between single-subject $\operatorname{PLV}^{j}(t)$ and clusters that were obtained by the clustering algorithm applied to $\overline{\operatorname{PLV}}(\mathrm{t})$. Again, each time point was labeled according to the graph with which it was best correlated, yielding a measure of graph existence. This procedure was particularly helpful to analyze, extract, and identify the spatiotemporal behaviors that are common across subjects.

\section{Data}

Six subjects were shown pictures on a screen using E-Prime 2.0 software (Psychology Software Tools, Pittsburgh, PA). They were requested to name the displayed pictures. The 148 images were selected from a database of 400 pictures standardized for French (Alario and Ferrand 1999) and were used during two sessions (about eight minutes each) of 74 stimuli. Brain activity was recorded using hr-EEG system (EGI, Electrical Geodesic Inc.). EEG signals were collected with a $1 \mathrm{kHz}$ sampling frequency and band-pass filtered between 3 and 45Hz. Each trial was visually inspected, and epochs contaminated by eye blinking, movements or any other noise source were rejected and excluded from the analysis. This study was approved by the National Ethics Committee for the Protection of Persons (CPP), 
(conneXion study, agreement number 2012-A01227-36, promoter: Rennes University Hospital). We excluded the electrodes located on the face as well as the few electrodes showing too high impedance. All participants provided a written informed consent to participate in this study.

\section{Results}

The algorithm described above was applied on the adjacency matrix averaged over 6 subjects who performed the picture recognition and naming task. The segmentation algorithm, applied to the recorded EEG signals, led to a number of clusters equal to 6 . Results are shown in figure 2A. The first cluster corresponded to the period ranging from $t=0$ (Stimulus onset: presentation of the picture) to $\mathrm{t}=116 \mathrm{~ms}$. In this cluster, a network located in the occipital lobe could be observed. A shorter graph was then observed between $\mathrm{t}=117 \mathrm{~ms}$ and $\mathrm{t}=153 \mathrm{~ms}$ with strong connections at the occipital lobe as well. The $3^{\text {rd }}$ cluster corresponded to the time interval 154-190 ms where connections appeared mainly in the temporal and parietal lobes. A network was then identified in the cluster 191-316 ms with the presence of connections mainly located in the frontal lobe, followed by a cluster (317-480 ms) with two separate networks in frontal and occipital lobes. The last cluster (481-620 ms) was then associated with a denser network in left temporal and bilateral frontal lobes with predominance of the right frontal lobe.

Figure 2B shows the results obtained regarding the inter-subject variability. The graph existence percentages were computed for all the subjects. The figure shows that graphs (T3, T6) have the highest graph existence ( $73 \%$ and $78 \%$ respectively). In contrast, the lowest values $(30.99 \%, 22.33 \%)$ were obtained for graph $\mathrm{T} 1$ and $\mathrm{T} 4$ respectively. The graphs $\mathrm{T} 2$ and T5 showed nearly similar graph existence with $41.17 \%$ and $38.33 \%$ respectively. Results also showed that the first (T1) and the fifth (T5) graphs have the lowest variability across subjects 
( $\pm 16 \%$ and $\pm 22 \%$ respectively) while the second graph (T2) has the highest variability $( \pm 38 \%)$

A

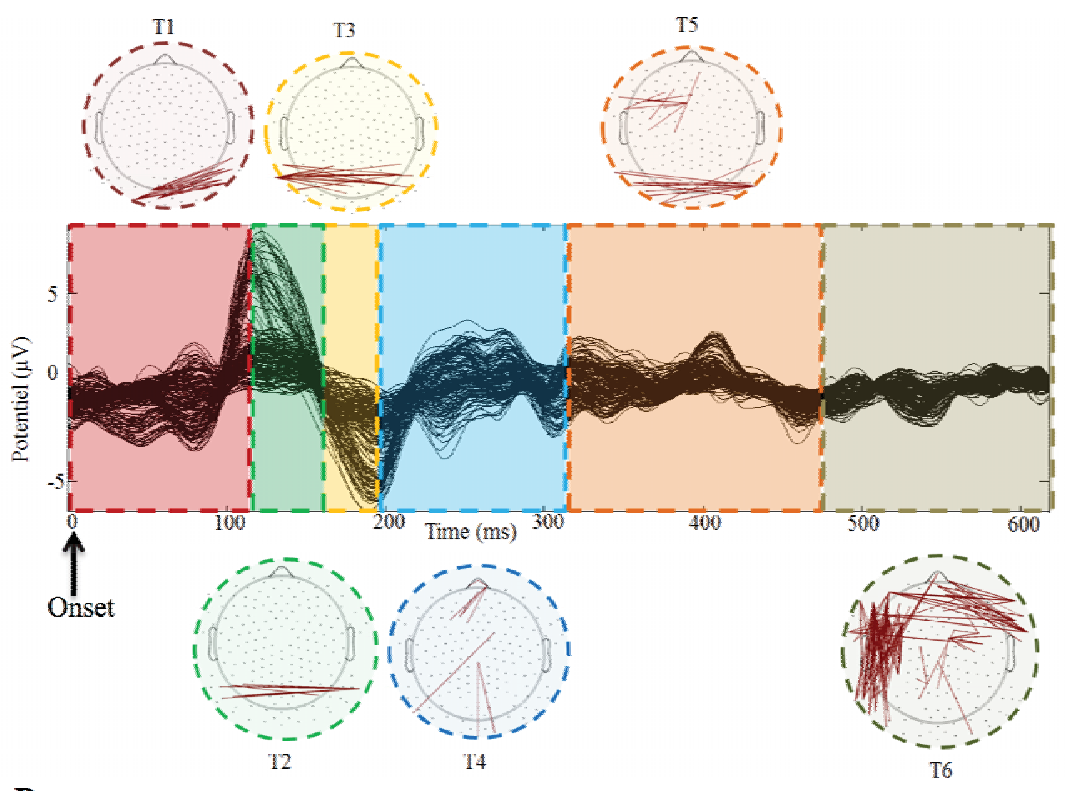

B

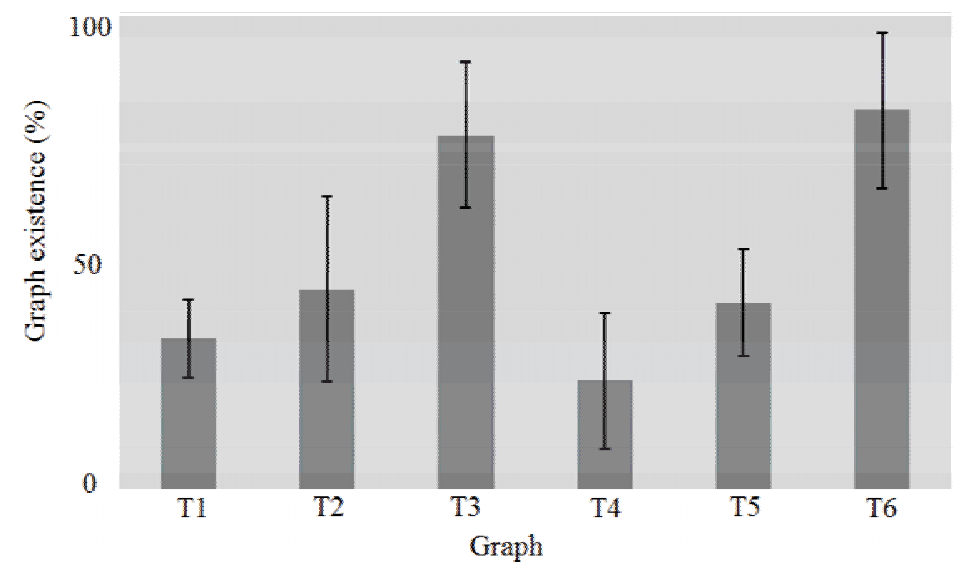

Figure 2: Results of the segmentation process A-: Event related potentials for the picture naming task and distribution of the functional connectivity graphs revealed by the proposed spatiotemporal segmentation algorithm. The results are shown from $0 \mathrm{~ms}$ (the Onset when the visual stimuli were presented) to $620 \mathrm{~ms}$ when the naming process starts. B- Graph existence is computed over the six subjects for the six identified networks. 


\section{Discussion and conclusion}

A challenge of cognitive neuroscience is to temporally follow, over very short time duration, the sequence of brain processes involved in a considered task.

In this communication, a novel algorithm was presented to track the dynamics of functional connectivity. The proposed algorithm is based on the clustering of connectivity matrices computed from the phase locking value method applied on scalp dense EEG signals. It is exemplified in the well-known picture naming task which duration is about of $500 \mathrm{~ms}$. By taking advantage of the excellent temporal resolution of the EEG signals and the use of PLV, the main originality of the proposed algorithm is its capacity to track connectivity dynamics over very short time periods. The proposed algorithm showed high performance to reveal the stability of the brain networks over short periods of time and to segment the cognitive process into functional connectivity microstates.

Another algorithm was recently proposed by Allen et al. (Allen, Damaraju et al. 2012) and applied to track brain networks during resting state. The basic principle of this algorithm was based on the independent component analysis and the k-means. However the proposed algorithm was adapted to fMRI data by taking a large time window (about 3s) to compute the functional connectivity and therefore does not allow tracking cognitive tasks with short time duration. Therefore, it is likely that accurate tracking of the picture naming could not be achieved with the above mentioned proposed algorithm.

Very interestingly, results were qualitatively consistent, in term of involved brain regions, with already-reported results regarding the analyzed task, most of them being however based on other neuroimaging modalities (mainly functional magnetic resonance-fMRI- and Positron Emission Tomography-PET-). Networks were identified in the occipital lobe during the first $200 \mathrm{~ms}$ (including the P150) which likely correspond to the visual processing of the presented picture during the recognition phase (Schendan, Ganis et al. 1998). Another network is then 
identified in the following $200 \mathrm{~ms}$ where a left occipito-parieto-temporal network was identified which is compatible with brain regions involved in the access to semantics content of the presented picture (Salmelin, Hari et al. 1994). Finally, at the last $200 \mathrm{~ms}$, dense networks appear in the temporo-frontal regions. It is well established that this network is strongly involved in decision making during the naming phase (Salmelin, Hari et al. 1994).

Regarding the EEG connectivity studies, our results seem to agree with studies reported the dynamic (time-varying) behavior of functional connectivity graphs during visual tasks (Babiloni, Cincotti et al. 2005; De Vico Fallani, Astolfi et al. 2008). These studies and others showed how the brain networks properties modify during visual tasks with relatively short duration.

The proposed method was applied to networks obtained at the level of scalp EEG electrodes. These networks suffer from the low spatial resolution and the effect of the of field spread. Our further objective is to apply this algorithm on EEG source connectivity graphs to identify the dynamics of brain networks at cortical source level as described in (Hassan, Dufor et al. 2014). The algorithm will be used also to compare the brain connectivity dynamics at different conditions such as the difference between networks related to different type of stimulus (animals vs. tools for instance).

More generally, the proposed algorithm can be broadly applicable to disciplines where network dynamics is crucial in the understanding of system performance.

\section{Acknowledgements}


This work has received a French government support granted to the CominLabs excellence laboratory and managed by the National Research Agency in the "Investing for the Future" program under reference ANR-10-LABX-07-01. It was also supported by the Rennes University Hospital (COREC Project named conneXion, 2012-14). We also thank the European Research Council for the ERC-2011-ADG - Grant Agreement No 290901 Acronym "NEUCOD".

\section{References}

Alario, F. X. and L. Ferrand (1999). "A set of 400 pictures standardized for French: Norms for name agreement, image agreement, familiarity, visual complexity, image variability, and age of acquisition." Behavior Research Methods 31(3): 531-552.

Allen, E. A., E. Damaraju, et al. (2012). "Tracking whole-brain connectivity dynamics in the resting state." Cerebral Cortex: bhs352.

Babiloni, F., F. Cincotti, et al. (2005). "Estimation of the cortical functional connectivity with the multimodal integration of high-resolution EEG and fMRI data by directed transfer function." Neuroimage 24(1): 118-131.

Bartolomei, F., I. Bosma, et al. (2006). "How do brain tumors alter functional connectivity? A magnetoencephalography study." Annals of neurology 59(1): 128-138.

De Vico Fallani, F., L. Astolfi, et al. (2008). "Brain network analysis from high-resolution EEG recordings by the application of theoretical graph indexes." Neural Systems and Rehabilitation Engineering, IEEE Transactions on 16(5): 442-452.

Dimitriadis, S. I., N. A. Laskaris, et al. (2010). "Tracking brain dynamics via time-dependent network analysis." Journal of neuroscience methods 193(1): 145-155.

Friston, K. J. (1994). "Functional and effective connectivity in neuroimaging: a synthesis." Hum Brain Mapp

\section{2(1-2): 56-78.}

Hassan, M., O. Dufor, et al. (2014). "EEG Source Connectivity Analysis: From Dense Array Recordings to Brain Networks." PloS one 9(8): e105041.

Lachaux, J.-P., E. Rodriguez, et al. (1999). "Measuring phase synchrony in brain signals." Human brain mapping 8(4): 194-208.

Murray, M. M., D. Brunet, et al. (2008). "Topographic ERP analyses: a step-by-step tutorial review." Brain Topogr 20(4): 249-264.

Rodriguez, E., N. George, et al. (1999). "Perception's shadow: long-distance synchronization of human brain activity." Nature 397(6718): 430-433.

Salmelin, R., R. Hari, et al. (1994). "Dynamics of brain activation during picture naming." Nature 368(6470): 463-465.

Schendan, H. E., G. Ganis, et al. (1998). "Neurophysiological evidence for visual perceptual categorization of words and faces within 150 ms." Psychophysiology 35(3): 240-251.

Sporns, O. (2010). Networks of the Brain, MIT Press. 